\title{
Spin structure of spin-orbit split surface states in a magnetic material revealed by spin-integrated photoemission
}

\author{
D. Yu. Usachov $\odot,{ }^{1, *}$ M. Güttler $\odot,{ }^{2}$ S. Schulz $\odot,{ }^{2}$ G. Poelchen $\odot,{ }^{2}$ S. Seiro, ${ }^{3}$ K. Kliemt $\odot,{ }^{4}$ K. Kummer $\odot,{ }^{5}$ C. Krellner $\odot,{ }^{4}$ \\ C. Laubschat, ${ }^{2}$ E. V. Chulkov, ${ }^{1,6,7,8,9}$ and D. V. Vyalikh $\oplus^{6,10}$ \\ ${ }^{1}$ St. Petersburg State University, 7/9 Universitetskaya nab., St. Petersburg 199034, Russia \\ ${ }^{2}$ Institut für Festkörper- und Materialphysik, Technische Universität Dresden, D-01062 Dresden, Germany \\ ${ }^{3}$ Leibniz IFW Dresden, Helmholtzstr. 20, D-01069 Dresden, Germany \\ ${ }^{4}$ Kristall- und Materiallabor, Physikalisches Institut, Goethe-Universität Frankfurt, \\ Max-von-Laue Strasse 1, D-60438 Frankfurt am Main, Germany \\ ${ }^{5}$ European Synchrotron Radiation Facility, 71 Avenue des Martyrs, Grenoble, France \\ ${ }^{6}$ Donostia International Physics Center (DIPC), 20018 Donostia/San Sebastián, Basque Country, Spain \\ ${ }^{7}$ Departamento de Física de Materiales UPV/EHU, 20080 Donostia/San Sebastián, Basque Country, Spain \\ ${ }^{8}$ Centro de Física de Materiales CFM-MPC and Centro Mixto CSIC-UPV/EHU, 20018 Donostia/San Sebastián, Basque Country, Spain \\ ${ }^{9}$ Tomsk State University, Lenina Av. 36, 634050 Tomsk, Russia \\ ${ }^{10}$ IKERBASQUE, Basque Foundation for Science, 48013 Bilbao, Spain
}

(Received 11 March 2020; revised manuscript received 3 May 2020; accepted 7 May 2020; published 15 June 2020)

\begin{abstract}
The emergence of ferromagnetism in Rashba systems, where the evolving exchange interaction enters into competition with spin-orbit coupling, leads to a nontrivial spin-polarized electronic landscape with an intricate momentum-dependent spin structure, which is challenging to unveil. Here, we show a way to disentangle the contributions from the effective spin-orbit and exchange fields and thus to gain knowledge of the spin structure in ferromagnetic Rashba materials, which is required for spintronic applications. Our approach is based exclusively on spin-integrated photoemission measurements combined with a two-band modeling. As an example, we consider the mixed-valent material $\mathrm{EuIr}_{2} \mathrm{Si}_{2}$ which, while being nonmagnetic in the bulk, reveals strong ferromagnetism at the iridium-silicide surface where both spin-orbit and exchange magnetic interactions coexist. The combined effect of these interactions causes a complex band dispersion of the surface state which can be observed in photoemission experiments. Our method allows us to comprehensively unravel the surface-state spin structure driven by spin-orbit coupling at the ferromagnetic surface. This approach opens up opportunities to characterize the spin structure of ferromagnetic Rashba materials, especially where dedicated spin-resolved measurements remain challenging.
\end{abstract}

DOI: 10.1103/PhysRevB.101.245140

\section{INTRODUCTION}

The successful development of spintronic applications, where the electron spin plays the key role, requires the elaboration of novel magnetically active materials with tunable spin-dependent properties [1,2]. There are several fundamental interactions which can be used to create a magnetically ordered state and to efficiently manipulate charge and spin currents in crystalline systems. The most involved is spin-orbit (SO) coupling, which in conjunction with a lack of inversion symmetry provides a spin-dependent potential at the surface or interface, leading to the so-called Rashba effect $[3,4]$.

The SO interaction can be further combined with exchange magnetic coupling, which often results in exotic electronic properties. For example, insertion of magnetic transition-metal (TM) layers into a topological insulator allows us to open a gap in the spin-polarized topological state,

*dmitry.usachov@spbu.ru providing suitable conditions for such phenomena as the quantum anomalous Hall effect or the emergence of chiral Majorana fermions [5]. The introduction of exchange interaction into TM dichalcogenides by means of the proximity effect provides the ability to control both the spin and valley degrees of freedom by choosing an appropriate ferromagnetic (FM) substrate, which breaks the symmetry between the two valleys [6]. Moreover, the proximity of magnetic and heavy atoms may induce notable SO and exchange effects in graphene, where these interactions are initially negligible [7].

Strong competition between magnetism and SO coupling can be achieved by inserting, for example, layers of a rareearth element with magnetically active $4 f$ moments into a matrix with heavy TM atoms. Moreover, the strength and orientation of the emerging exchange field can also be adjusted by rotation of the $4 f$ moments through their coupling to a crystal-electric field. The resulting effective magnetic field, which originates from the SO and exchange interactions, provides a degree of freedom to manipulate the electron spin. Furthermore, the Kondo interaction, when combined with the 
Rashba effect, also allows us to finely tune the spin-dependent properties of two-dimensional electrons [8]. Thus, mutual combinations of these interactions give many possibilities to manipulate the magnetic properties of a system in unusual ways, while at the same time its spin structure can be rather complex, providing an interesting subject for experimental studies [9]. Nevertheless, studies of the experimental band and spin structure of the discussed systems remain rather scarce. Theory predicts asymmetric distortions of the electronic bands under the influence of an exchange field when it is not orthogonal to the SO field [10]. Such distortions were indeed observed for a Rashba-split surface state on a FM surface [11]. Orthogonal orientation of the two effective fields results in an opening of Zeeman gaps, as was observed in ferroelectric crystals with magnetic impurities [12].

Respective studies of the spin structure pose certain challenges due to a number of factors. The most direct way to gain insight into the momentum-resolved properties of electron spins is spin- and angle-resolved photoelectron spectroscopy (SR-ARPES) [13]. With this method, difficulties arise from the choice of the geometry of the experiment and the resulting matrix element effects which should be accurately considered when conclusions on the spin expectation value in the ground state are derived [14-16]. To this end, it is useful to evaluate the experimental results by comparison to ab initio calculations of the spin-polarized photocurrent performed in the frame of a one-step theory of photoemission, which, however, is a difficult task. Another difficulty is linked with the preparation of a clean and appropriate sample surface, which may be formed by different terminations and magnetic domains. The structure of such domains and the boundaries between them can strongly affect the SR-ARPES measurements and impede an accurate interpretation of the results. A solution of this problem requires high spatial resolution, which significantly complicates spin-resolved measurements.

In this paper, we show that the information on the spin structure of a magnetic surface can be obtained from conventional ARPES measurements. However, for the successful application of the proposed approach, it is necessary that both SO coupling and exchange magnetism are present in the system. To demonstrate our approach, we consider the magnetic iridium-silicide surface of the mixed-valent material $\mathrm{EuIr}_{2} \mathrm{Si}_{2}$ [9]. Our analysis shows that information on the spin structure of surface states derived from conventional ARPES measurements is rather similar to that obtained from SRARPES measurements complemented by calculations of the spin-polarized photocurrent [16].

\section{METHODS}

High-quality single crystalline samples of $\mathrm{EuIr}_{2} \mathrm{Si}_{2}$ were cleaved in situ under ultrahigh vacuum conditions at low temperature. The obtained surface exhibited large (up to several hundreds of microns in size) magnetic domains, which were easily identified by ARPES due to different (rotated) band structures of the studied surface states [9]. The ARPES experiments were performed at the I05 beamline of the Diamond Light Source. Photoemission data for the paramagnetic (PM) and FM phases were obtained at temperatures of $200 \mathrm{~K}$ and $7 \mathrm{~K}$, respectively.
Fully relativistic density functional theory (DFT) calculations of the electronic and spin structures of $\mathrm{EuIr}_{2} \mathrm{Si}_{2}$ were performed using FPLO code [17]. Further details can be found in the Supplemental Material (SM) [18].

\section{RESULTS AND DISCUSSION}

In many cases, a simplified approach considering one electron, which is moving in the mean field produced by the other particles, is sufficient to describe the most essential properties of a studied material. For a FM material with SO coupling, the one-particle Hamiltonian can be written as

$$
\hat{H}=\frac{\hat{\mathbf{p}}^{2}}{2 m}+V+\frac{\hbar}{4 m^{2} c^{2}}(\nabla V \times \hat{\mathbf{p}}) \cdot \hat{\boldsymbol{\sigma}}+J_{\mathrm{ex}} \mathbf{M} \cdot \hat{\boldsymbol{\sigma}},
$$

where $\hat{\mathbf{p}}$ is the momentum operator, $V$ the one-electron potential, $\hat{\sigma}$ the operator of the Pauli matrices, $J_{\text {ex }}$ the exchange coupling strength in presence of the magnetization $\mathbf{M}$, and $\nabla V$ the effective local electric field. The third term on the right side represents SO coupling, while the fourth term describes the exchange coupling of the electron spin with the spins of the other electrons. The simplest model describing an electron band, which is spin split due to SO or exchange interaction, is based on the following two-band Hamiltonian:

$$
\hat{H}(\mathbf{k})=U(\mathbf{k}) \cdot \sigma_{0}+\mathbf{B}(\mathbf{k}) \cdot \hat{\boldsymbol{\sigma}}-\mathbf{J} \cdot \hat{\boldsymbol{\sigma}},
$$

where $\mathbf{B}(\mathbf{k})$ and $\mathbf{J}$ can be understood as effective SO and exchange fields, respectively. The corresponding band dispersions are given by

$$
E^{ \pm}(\mathbf{k})=U(\mathbf{k}) \pm|\mathbf{B}(\mathbf{k})-\mathbf{J}|,
$$

where $U(\mathbf{k})$ is the dispersion in absence of SO and exchange coupling. The term $\mathbf{B}(\mathbf{k})-\mathbf{J}$, which is a total effective field, describes the magnitude of the spin splitting of the band and determines the direction of the electron spin. In the following, we demonstrate that, under certain conditions, it is possible to determine the effective fields, and thus the spin structure of the electron band directly from the dispersions $E^{ \pm}(\mathbf{k})$ measured in a conventional ARPES experiment.

To demonstrate our approach, we consider the mixedvalent material $\mathrm{EuIr}_{2} \mathrm{Si}_{2}$ discovered in 1986 [19]. It was shown to be nonmagnetic in the bulk, but strongly FM at the (001) surface [9]. In the bulk, the Eu atoms are in a valencefluctuating state down to lowest temperatures [19-21]. However, at the iridium-silicide surface, represented by a Si-IrSi-Eu (SISE) surface block, Eu exhibits a magnetic divalent state. The $4 f$ magnetic moments of this divalent Eu layer order ferromagnetically below $48 \mathrm{~K}$ [9]. This results in a large exchange polarization of the electrons, which occupy the surface states hosted by the topmost SISE block. However, because heavy Ir atoms are strongly involved in these surface states, the absence of inversion symmetry at the surface results in a large spin splitting of the surface states already in the PM phase, i.e., above $48 \mathrm{~K}$, due to the Rashba effect. Thus, when the temperature is lowered, the emerging in-plane exchangemagnetic field starts to act together with the SO field on the surface-state electrons. This leads to a strong distortion of the Fermi contours and electron band dispersions when the magnetization is oriented in plane [11]. In the studied system, the $4 f$ magnetic moments of Eu in the fourth layer below the 


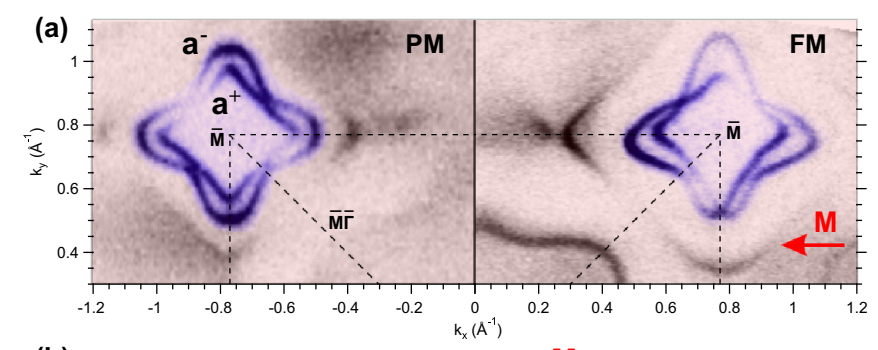

(b)

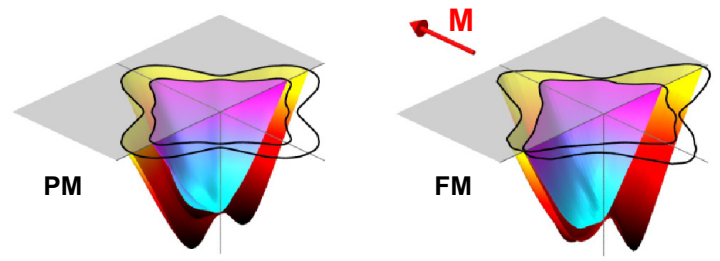

FIG. 1. ARPES view on the Fermi surface with the surface state highlighted in blue (a) and its electron band dispersions $E(\mathbf{k})$ (b) around the $\bar{M}$ point for paramagnetic and ferromagnetic Siterminated surfaces of $\mathrm{EuIr}_{2} \mathrm{Si}_{2}$. Dashed lines indicate the border of the surface Brillouin zone. The red arrow indicates the direction of magnetization which stems from ordered $\mathrm{Eu} 4 f$ moments.

SISE surface block are parallel to the surface, similar to the $\mathrm{EuRh}_{2} \mathrm{Si}_{2}$ or $\mathrm{GdRh}_{2} \mathrm{Si}_{2}$ systems [22,23]. The signature of the out-of-plane component would be the sizable energy gap at the $\bar{M}$ point, similar to the $\mathrm{HoRh}_{2} \mathrm{Si}_{2}$ system [24].

We illustrate this phenomenon in Fig. 1. The left panel of Fig. 1(a) shows the ARPES view on one of the mentioned surface states at a temperature of $200 \mathrm{~K}$, when the $\mathrm{Eu} 4 f$ magnetic moments are disordered and the surface is in the PM phase $(J \approx 0)$. Due to the Rashba effect, the Fermi contour reveals a spin splitting in two bands marked as $\alpha^{+}$and $\alpha^{-}$. They both reflect the fourfold symmetry of the crystal. When the Eu $4 f$ moments become ordered and the surface undergoes a transition to the FM state, the evolving magnetization M strives to break up the spin-momentum locking induced by SO coupling. This leads to a strong rescaling of the binding energies, distortion of the Fermi contour and reduction of rotational symmetry as one can see in the right panel of Fig. 1(a). It should be noted that these ARPES data were obtained from a single magnetic domain [9].

The appearance of asymmetry in the dispersions is also illustrated in Fig. 1(b). It has a clear physical meaning: When the effective SO field is parallel or antiparallel to the emerging exchange field $\mathbf{J}$ the energy splitting of the band gets smaller or larger, respectively. This implies that the band distortion observed in the FM phase reveals the direction of the SO field. Although the effective SO field in the FM phase may slightly differ from that in the PM phase [25], we assume them to be equal in our model $\left(\mathbf{B}_{\mathrm{FM}}=\mathbf{B}_{\mathrm{PM}}=\mathbf{B}\right)$. Under this assumption, the band distortion in the FM phase can be directly related to the spin orientation in the PM phase. Thus, from the band dispersions measured in the FM phase, one can reconstruct the spin structure for the PM phase. Note that for the realization of this approach, we need only conventional ARPES measurements. Let us consider this idea quantitatively.

The time-reversal symmetry in the PM phase requires that $\mathbf{B}(-\mathbf{k})=-\mathbf{B}(\mathbf{k})$. Using this fact, from Eq. (3) it is easy to
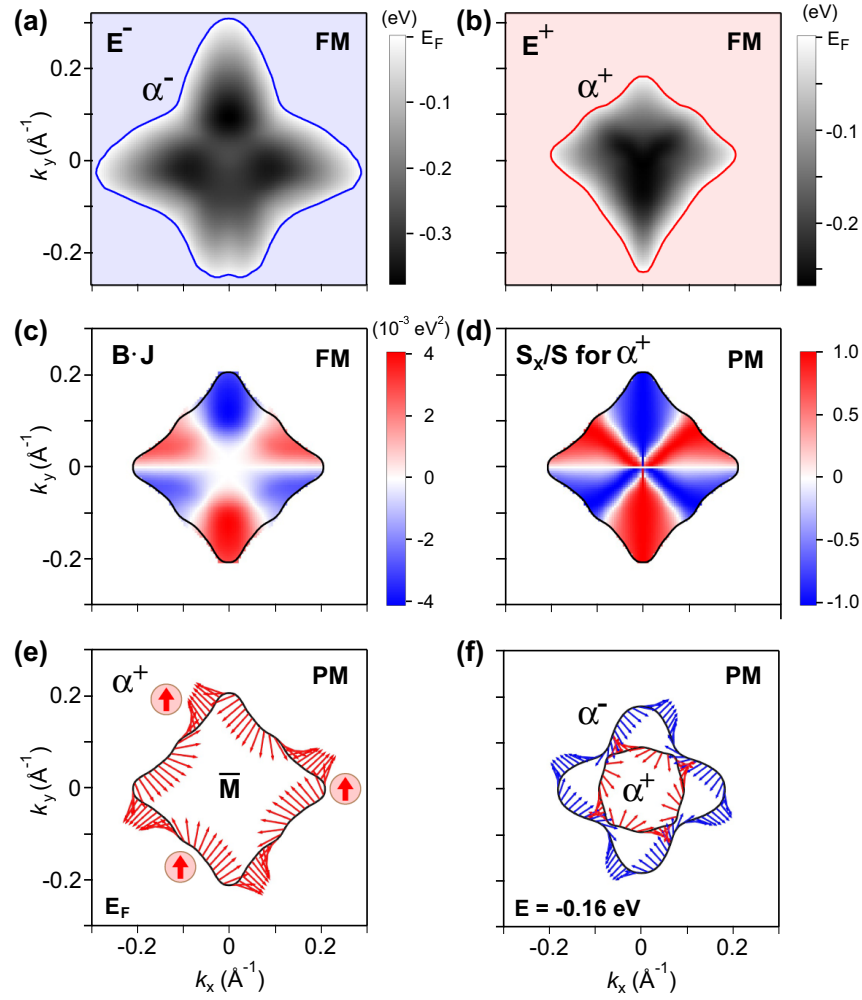

FIG. 2. (a), (b) Measured Fermi surfaces and band dispersions $E^{ \pm}(\mathbf{k})$ in the FM phase. (c) $\mathbf{B} \cdot \mathbf{J}$ product, showing a triple winding of the effective SO field. (d) Normalized $S_{x}$ component of spin for the $\alpha^{+}$state in the PM phase. (e), (f) Experimentally obtained spin structure in the PM phase (e) at the Fermi level and (f) at the binding energy of $0.16 \mathrm{eV}$. Here and further, the $k$ values are measured relative to the $\bar{M}$ point.

obtain the formula expressing the scalar product $\mathbf{B} \cdot \mathbf{J}$ through the energy splitting $\Delta$ of the band of interest (see details in the SM [18]),

$$
\mathbf{B} \cdot \mathbf{J}=\frac{\Delta^{2}(-\mathbf{k})-\Delta^{2}(\mathbf{k})}{16},
$$

where $\Delta(\mathbf{k})=E^{+}(\mathbf{k})-E^{-}(\mathbf{k})$ (see Fig. S1 in the SM [18]) and $-\mathbf{k}=\left(-k_{x},-k_{y}\right)$. This expression is not the only possible one, but in our opinion it is most convenient for the analysis of ARPES data. Figures 2(a) and 2(b) show the experimentally obtained binding energies and the Fermi contours of the discussed surface state for the FM phase. To improve the quality of results, we used an apparent symmetry of the measured dispersions: $E\left(k_{x}, k_{y}\right)=E\left(-k_{x}, k_{y}\right)$. The product B $\cdot \mathbf{J}$, obtained from the measured dispersions of the $\alpha^{ \pm}$states using Eq. (4), is shown in Fig. 2(c). It immediately reveals three directions in $k$ space, seen as white stripes, where $\mathbf{B} \cdot \mathbf{J}=0$, i.e., $\mathbf{B}$ and also the spin vector in the PM phase becomes orthogonal to $\mathbf{J}$.

Thus, using the ARPES data for the FM phase, it is possible to obtain one component of the vector $\mathbf{W}=\mathbf{B} J$, which describes an effective SO field scaled by the absolute value of J. Its second component can possibly be obtained from symmetry considerations. However, to determine the vector $\mathbf{B}$, one needs to know the direction of $\mathbf{J}$. Since $\mathbf{J}=-J_{\mathrm{ex}} \mathbf{M}$, 

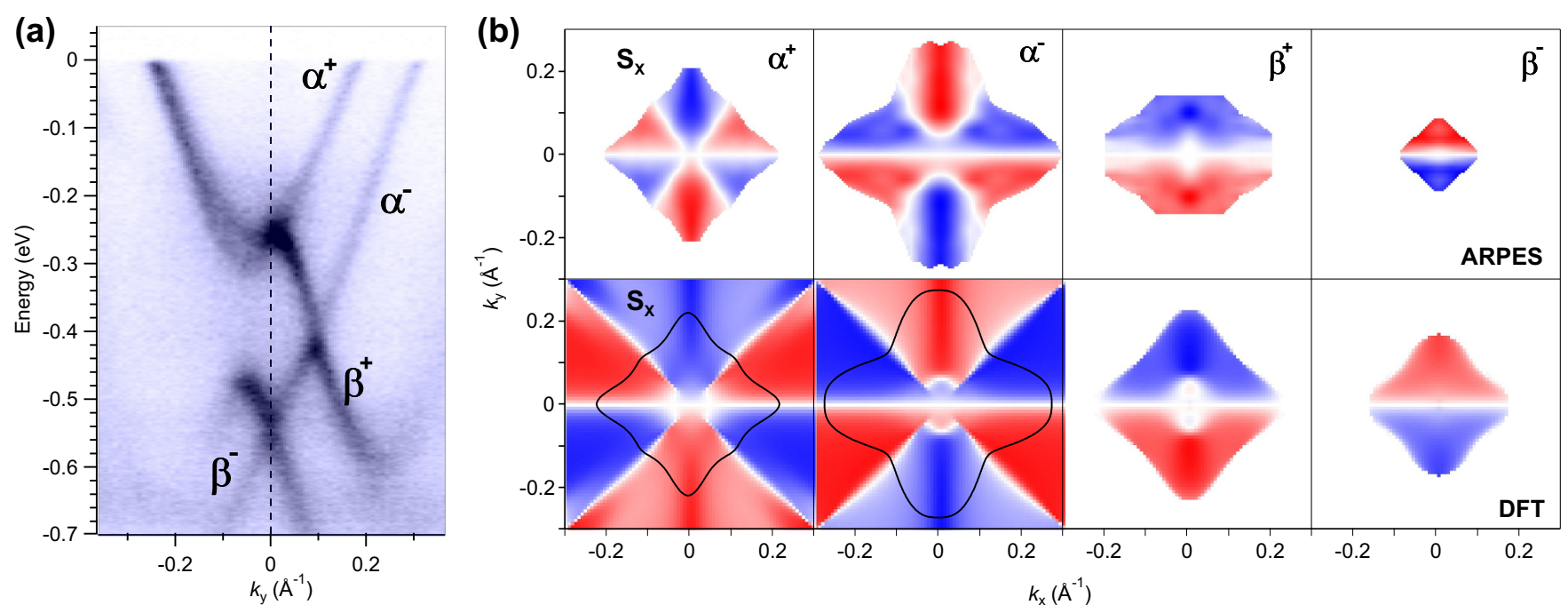

FIG. 3. (a) The ARPES data for the FM phase of $\operatorname{EuIr}_{2} \mathrm{Si}_{2}$ measured along the $y$ axis in Fig. 1(a) through the $\bar{M}$ point $(\bar{X} \bar{M} \bar{X}$ direction). (b) The spin component $S_{x}$ in the PM phase, qualitatively determined from the ARPES data as $S_{x}^{ \pm} \propto \pm B_{x}$ using Eq. (9), compared with the spin component $S_{x}$, derived from the DFT calculations. Solid lines indicate Fermi contours.

the exchange field can either be parallel or antiparallel to the magnetization, depending on the sign of the exchange coupling parameter $J_{\mathrm{ex}}$. The direction of the magnetization can be determined in the photoemission experiment using, e.g., circular dichroism in the $4 f$ photoemission spectrum [26]. However, in this paper, we determined it by comparing the measured band structure with the calculated one [9]. In the case of our $\mathrm{EuIr}_{2} \mathrm{Si}_{2}$ sample, the orientation of the $\mathbf{M}$ vector is shown in Fig. 1. From the DFT calculations (see SM [18]), we find that the energy of the states having $S_{x}>0$ is decreased when the exchange interaction is switched on. This means that $J_{\text {ex }}>0$. Hence, in our case, the exchange field $\mathbf{J}$ is oriented along the $x$ axis (the [100] crystallographic direction), and we get

$$
W_{x}(\mathbf{k})=\mathbf{B} \cdot \mathbf{J} .
$$

Due to the fourfold symmetry of the crystal, one can readily find the second $(y)$ component of $\mathbf{W}$ by rotating the $W_{x}$ component by $90^{\circ}$ :

$$
W_{y}\left(k_{x}, k_{y}\right)=W_{x}\left(k_{y},-k_{x}\right)
$$

Presence of the fourfold symmetry and time-reversal symmetry also requires that $W_{z}=0$ in our system. Thus, there is no out-of-plane spin component in the PM phase in the studied system (note that this may not be the case for surfaces with a threefold symmetry $[27,28]$ ). As a result, from the dispersions measured for the FM phase, we can determine all components of the vector $\mathbf{W}$, which indicates the direction of the SO field. This allows us to determine the direction of the spin vector in the PM phase as a function of $\mathbf{k}$ because the spin vector is oriented along the SO field. In particular,

$$
\frac{\mathbf{S}_{\mathrm{PM}}^{ \pm}(\mathbf{k})}{S_{\mathrm{PM}}^{ \pm}(\mathbf{k})}= \pm \frac{\mathbf{B}(\mathbf{k})}{B(\mathbf{k})}= \pm \frac{\mathbf{W}(\mathbf{k})}{W(\mathbf{k})}
$$

The resulting spin structure is shown in Figs. 2(d) and 2(e) for the upper band $\alpha^{+}$. It should be noted that in the two-band model, the spin polarization is always $100 \%(S(\mathbf{k})=1)$, while it is not the case in real systems. For the considered surface states, the DFT calculation predicts $0.6 \leqslant S_{\mathrm{PM}}^{+} \leqslant 0.9$ at the Fermi level.

Since we are only able to determine $\mathbf{W}$ in a certain range of $k$ space [where ARPES data for Eq. (4) are available], the spin structure of the $\alpha^{-}$band can only be plotted at energies sufficiently below the Fermi level. The obtained spin structures of both bands are shown in Fig. 2(f). It reveals a triple winding of the spin vector when going along the constant energy contours that is related to the so-called cubic Rashba effect $[9,16,29]$. Recently, this type of spin structure was revealed by a spin-resolved ARPES study of the surface state in $\mathrm{TbRh}_{2} \mathrm{Si}_{2}$ [16], which belongs to the same $R T_{2} \mathrm{Si}_{2}$ family as $\mathrm{EuIr}_{2} \mathrm{Si}_{2}$, where $R$ and $T$ are rare-earth and TM atoms, respectively.

Let us consider a second equation for $\mathbf{B} \cdot \mathbf{J}$ which we express through the energies at opposite $\mathbf{k}$ points (see SM [18]):

$$
\mathbf{B} \cdot \mathbf{J}=\frac{E^{+}(-\mathbf{k})-E^{+}(\mathbf{k})}{2} \cdot \frac{E^{+}(-\mathbf{k})-E^{-}(\mathbf{k})}{2} .
$$

The energy difference between the bands at $\pm \mathbf{k}$ is less precisely determinable from the experimental data than the band splitting. However, the benefit of this equation in comparison to Eq. (4) is that the second factor in the right part is non-negative for any $\mathbf{k}$, for which the two-band model is appropriate. Thus, this factor can be omitted if one wishes to determine only the sign of $\mathbf{B} \cdot \mathbf{J}$.

If the absolute values of the SO and exchange fields obey the relation $B \ll J$ (which is not the case for the studied system), the discussed factor in Eq. (8) tends to $J$ and we obtain the projection of $\mathbf{B}$ on the direction of $\mathbf{J}$ as [25]

$$
B_{J}(\mathbf{k}) \approx \pm \frac{E^{ \pm}(-\mathbf{k})-E^{ \pm}(\mathbf{k})}{2}
$$

This expression involves only one band (any $E^{+}$and $E^{-}$ can be selected) and it can be used for qualitative analysis of the spin structure. Even when $B$ is rather large, Eq. (9) 
(a)

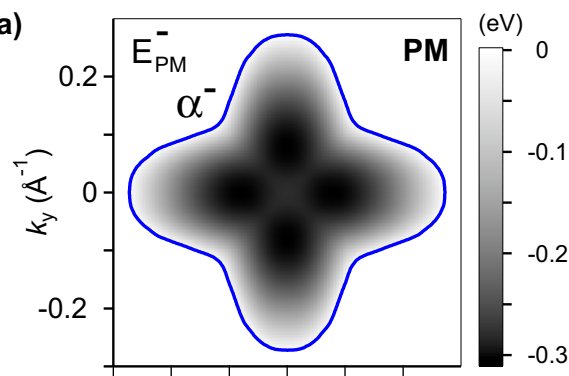

(d)

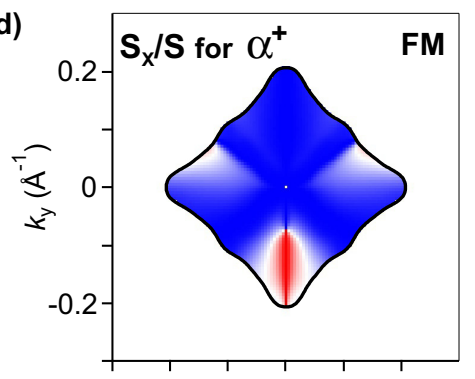

(g)

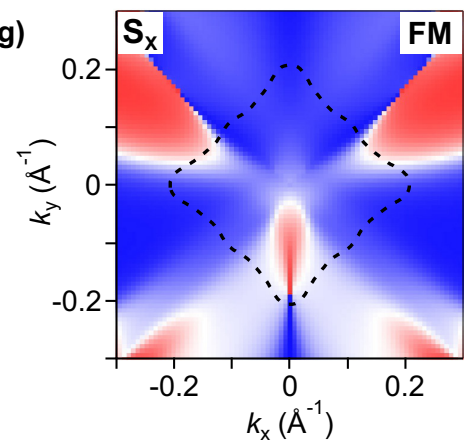

(b)

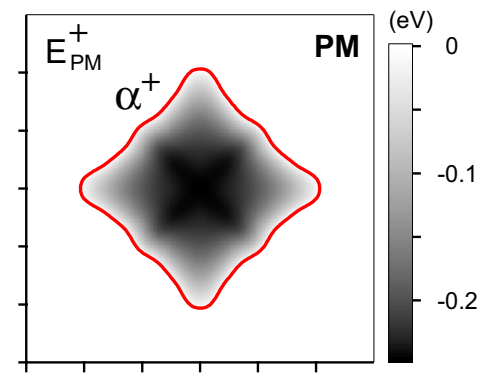

(e)

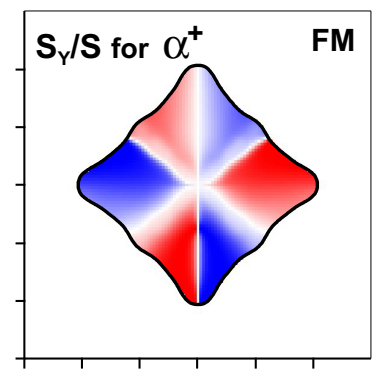

(h)

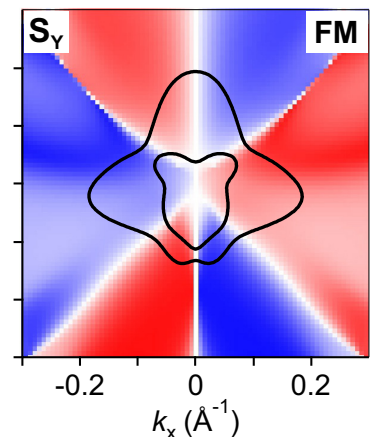

(c)

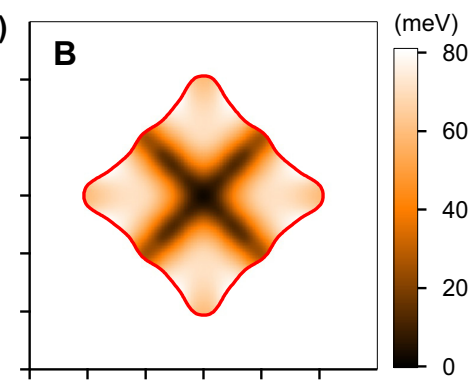

(f)

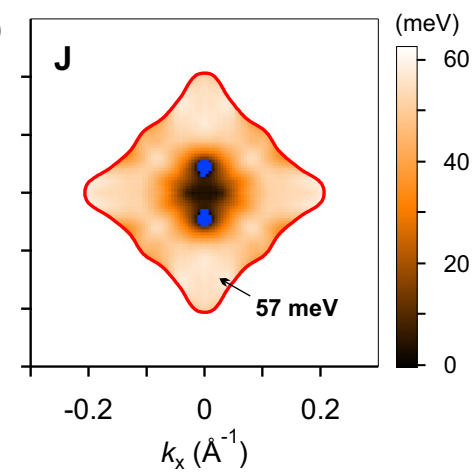

(i)

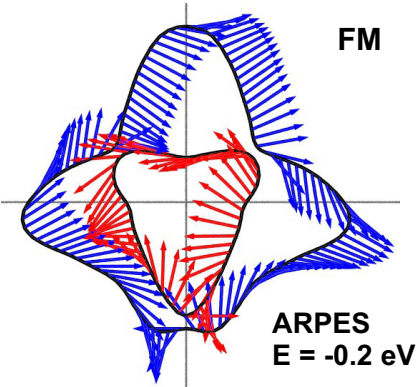

FIG. 4. (a), (b) Measured Fermi surfaces and dispersions for the $\alpha^{ \pm}$states in the PM phase. (c) The absolute value of the effective SO field obtained from the measured dispersion using Eq. (10). (d), (e) Spin components in the FM phase extracted from the ARPES data by means of Eq. (13). (f) The strength of exchange coupling derived from experiment using Eq. (11). Blue color indicates regions of undefined $J$. (g), (h) Spin expectation values for the $\alpha^{+}$band derived from the DFT calculation. Dashed line in (g) shows the measured Fermi contour in the PM phase, similar to the solid lines in (b)-(f). Solid lines in (h) show the calculated constant energy contours at $-0.2 \mathrm{eV}$ in the FM phase. (i) Spin texture obtained from ARPES with the use of Eq. (13).

identifies the sign of $B_{J}$ (as was discussed above) and reveals the $\mathbf{k}$ points where the spin vector (in the PM phase) becomes orthogonal to J. This is illustrated in Fig. 3 where Eq. (9) is applied to the four bands forming the two spin-split surface states $\alpha$ and $\beta$ [Fig. 3(a)]. In Fig. 3(b), one can recognize a very good agreement between the spin component $S_{x}$ determined from the ARPES data with the corresponding results from DFT calculations. It can be seen that in contrast to the $\alpha$ band, the $\beta$ band exhibits a spin structure that is rather similar to the classical Rashba-type spin structure with a single winding of the spin vector around the $\bar{M}$ point.

Let us now turn to the analysis of the spin structure in the FM phase. To derive it, the absolute values $B$ and $J$ are required. The former can be obtained from the band splitting measured in the PM phase, when $J=0$ :

$$
B(\mathbf{k})=\frac{E_{\mathrm{PM}}^{+}(\mathbf{k})-E_{\mathrm{PM}}^{-}(\mathbf{k})}{2} .
$$

Figures 4(a) and 4(b) show the dispersions of the $\alpha^{+}$and $\alpha^{-}$states obtained with ARPES in the PM phase, while
Fig. 4(c) displays the corresponding function $B(\mathbf{k})$ obtained with Eq. (10). One can see that the strength of the effective SO field ranges from zero at the $\bar{M}$ point to $80 \mathrm{meV}$. Further, one can estimate $J$ as (see SM [18])

$$
J=\sqrt{\frac{\Delta^{2}(-\mathbf{k})+\Delta^{2}(\mathbf{k})}{8}-B^{2}(\mathbf{k})} .
$$

The right part of this expression is a function of $\mathbf{k}$; therefore, we can formally obtain a function $J(\mathbf{k})$, which is shown in Fig. 4(f). It can be seen that $J$ tends to zero in the vicinity of the $\bar{M}$ point. Moreover, there are regions where the expression under the square root is negative. These results indicate that the two-band model with a simple Zeeman term fails near the $\bar{M}$ point.

According to theoretical studies of the $\bar{M}$-surface state in $R T_{2} \mathrm{Si}_{2}$ materials, its spin polarization becomes hidden near the $\bar{M}$ point [29]. This results in vanishing spin expectation values and a tiny spin splitting in this region. These features can be described only by using a more sophisticated model Hamiltonian $[24,29]$. Thus, the central area with small $J$ in 
Fig. 4(f) shows the region where the spin polarization of the surface states vanishes. This is confirmed by DFT results for the polarization shown in Figs. S2(b) and S2(c) in the SM [18]. Sufficiently far from the $\bar{M}$ point, the function $J(\mathbf{k})$ has values of $50-60 \mathrm{meV}$. We have chosen a value of $57 \mathrm{meV}$ for the further analysis of the spin structure in the FM phase.

Once we have estimated $\mathbf{J}$, we can obtain $\mathbf{B}$ as

$$
\mathbf{B}(\mathbf{k})=B(\mathbf{k}) \frac{\mathbf{W}(\mathbf{k})}{W(\mathbf{k})}
$$

and derive the spin structure for the FM phase as

$$
\frac{\mathbf{S}_{\mathrm{FM}}^{ \pm}(\mathbf{k})}{S_{\mathrm{FM}}^{ \pm}(\mathbf{k})}= \pm \frac{\mathbf{B}(\mathbf{k})-\mathbf{J}}{|\mathbf{B}(\mathbf{k})-\mathbf{J}|} .
$$

The resulting spin components for the $\alpha$ band are shown in Figs. 4(d) and 4(e). They exhibit a good agreement with our DFT calculations of the spin expectation values, presented in Figs. 4(g) and 4(h). Thus, the validity of the proposed approach in the case of $\mathrm{EuIr}_{2} \mathrm{Si}_{2}$ is fully supported by theory. The ARPES-derived spin structure plotted along the constantenergy contours is shown in Fig. 4(i). One can see that the exchange interaction destroys the triple winding of the spin vector observed in the PM phase. While the $S_{y}$ component remains qualitatively similar to that in the PM case, the $S_{x}$ component is strongly affected, since the spin vector tends to align parallel or antiparallel to the exchange field $\mathbf{J}$.

At this stage, we should point out the conditions under which the proposed approach is expected to be efficient. It is essential that the magnetization should not be orthogonal to the surface of the studied crystal. The ARPES data must be collected from a single magnetic domain. The described approach is valid only in the regions of $\mathbf{k}$ space where the spin-split states can be well described by the simple twoband Hamiltonian with constant exchange coupling parameter $J_{\text {ex }}$. Regions with band anticrossings should be avoided. The analysis relies on the determination of the magnetization direction and on the knowledge of the sign of $J_{\mathrm{ex}}$. If the latter is unknown, the spin structure can be determined only with accuracy to the sign (direction) of the spin vector. The proposed approach allows to analyze only one component of the spin vector in the direction of $\mathbf{J}$, however, usage of the crystal symmetry can allow us to obtain the full spin structure of the studied surface states.

\section{CONCLUSIONS}

In summary, we have demonstrated a concept for determining the spin structure of electronic surface states, which experience both $\mathrm{SO}$ and magnetic exchange interactions. Our analysis of the band dispersions obtained from ARPES measurements allows us to gain detailed information on the spin structure and effective SO field. To illustrate our approach, we have used the nonmagnetic mixed-valent material $\mathrm{EuIr}_{2} \mathrm{Si}_{2}$, which shows pronounced FM properties at its iridium-silicide surface at low temperatures. In presence of an in-plane surface magnetization, the effective SO and exchange fields manifest themselves in a characteristic asymmetry of the surface state band dispersions. We show that the spin structure of such states in the PM phase can be deduced from ARPES measurements in the FM phase. Using a simple two-band model Hamiltonian, we revealed a rather complex spin structure with a triple winding of the spin vector at the Fermi contour. Combining the measurements in the PM and FM phases at the surface, we obtained a comprehensive description of the effective SO and exchange fields governing the spin structure of the surface states in both phases. The proposed approach can be a versatile tool for studying magnetic materials and systems where the SO-split surface states experience magnetic exchange interaction.

\section{ACKNOWLEDGMENTS}

This work was supported by Saint Petersburg State University (Grant No. ID 51126254) and the Russian Foundation for Basic Research (Grant No. 20-32-70127). We acknowledge financial support from the Spanish Ministry of Economy (No. MAT-2017-88374-P) and the German Research Foundation (DFG) through Grants No. LA655/20-1, No. KR3831/5-1, and Fermi-NEst. We acknowledge Diamond Light Source for access to beamline I05 (Proposals No. SI18844-1 and No. SI17761-1). We thank I. Nechaev for fruitful discussions.
[1] S. A. Wolf, D. D. Awschalom, R. A. Buhrman, J. M. Daughton, v. S. Molnar, M. L. Roukes, A. Y. Chtchelkanova, and D. M. Treger, Spintronics: A spin-based electronics vision for the future, Science 294, 1488 (2001).

[2] I. Žutić, J. Fabian, and S. D. Sarma, Spintronics: Fundamentals and applications, Rev. Mod. Phys. 76, 323 (2004).

[3] E. I. Rashba and V. I. Sheka, Symmetry of energy bands in crystals of wurtzite type II. Symmetry of bands with spin-orbit interaction included, Fiz. Tverd. Tela: Collected Pap. 2, 62 (1959).

[4] Y. A. Bychkov and E. I. Rashba, Properties of a 2D electron gas with lifted spectral degeneracy, JETP Lett. 39, 78 (1984).

[5] M. M. Otrokov, I. I. Klimovskikh, H. Bentmann, D. Estyunin, A. Zeugner, Z. S. Aliev, S. Gaß, A. U. B. Wolter, A. V. Koroleva, A. M. Shikin, M. Blanco-Rey, M. Hoffmann, I. P. Rusinov, A. Yu. Vyazovskaya, S. V. Eremeev, Yu. M.
Koroteev, V. M. Kuznetsov, F. Freyse, J. Sánchez-Barriga, I. R. Amiraslanov, M. B. Babanly, N. T. Mamedov, N. A. Abdullayev, V. N. Zverev, A. Alfonsov, V. Kataev, B. Büchner, E. F. Schwier, S. Kumar, A. Kimura, L. Petaccia, G. Di Santo, R. C. Vidal, S. Schatz, K. Kißner, M. Ünzelmann, C. H. Min, S. Moser, T. R. F. Peixoto, F. Reinert, A. Ernst, P. M. Echenique, A. Isaeva, and E. V. Chulkov, Prediction and observation of an antiferromagnetic topological insulator, Nature 576, 416 (2019).

[6] J. C. G. Henriques, G. Catarina, A. T. Costa, J. FernándezRossier, and N. M. R. Peres, Excitonic magneto-optical Kerr effect in two-dimensional transition metal dichalcogenides induced by spin proximity, Phys. Rev. B 101, 045408 (2020).

[7] A. G. Rybkin, A. A. Rybkina, M. M. Otrokov, O. Y. Vilkov, I. I. Klimovskikh, A. E. Petukhov, M. V. Filianina, V. Y. Voroshnin, I. P. Rusinov, A. Ernst, A. Arnau, E. V. Chulkov, and 
A. M. Shikin, Magneto-spin-orbit graphene: Interplay between exchange and spin-orbit couplings, Nano Lett. 18, 1564 (2018).

[8] A. Generalov, J. Falke, I. A. Nechaev, M. M. Otrokov, M. Güttler, A. Chikina, K. Kliemt, S. Seiro, K. Kummer, S. Danzenbächer, D. Usachov, T. K. Kim, P. Dudin, E. V. Chulkov, C. Laubschat, C. Geibel, C. Krellner, and D. V. Vyalikh, Strong spin-orbit coupling in the noncentrosymmetric Kondo lattice, Phys. Rev. B 98, 115157 (2018).

[9] S. Schulz, I. A. Nechaev, M. Güttler, G. Poelchen, A. Generalov, S. Danzenbächer, A. Chikina, S. Seiro, K. Kliemt, A. Yu. Vyazovskaya, T. K. Kim, P. Dudin, E. V. Chulkov, C. Laubschat, E. E. Krasovskii, C. Geibel, C. Krellner, K. Kummer, and D. V. Vyalikh, Emerging 2D-ferromagnetism and strong spin-orbit coupling at the surface of valence-fluctuating EuIr $_{2} \mathrm{Si}_{2}$, npj Quantum Mater. 4, 26 (2019).

[10] S. Grytsyuk, A. Belabbes, P. M. Haney, H.-W. Lee, K.-J. Lee, M. D. Stiles, U. Schwingenschlögl, and A. Manchon, $k$-asymmetric spin splitting at the interface between transition metal ferromagnets and heavy metals, Phys. Rev. B 93, 174421 (2016).

[11] O. Krupin, G. Bihlmayer, K. M. Döbrich, J. E. Prieto, K. Starke, S. Gorovikov, S. Blügel, S. Kevan, and G. Kaindl, Rashba effect at the surfaces of rare-earth metals and their monoxides, New J. Phys. 11, 013035 (2009).

[12] J. Krempaský, S. Muff, F. Bisti, M. Fanciulli, H. Volfová, A. P. Weber, N. Pilet, P. Warnicke, H. Ebert, J. Braun, F. Bertran, V. V. Volobuev, J. Minár, G. Springholz, J. H. Dil, and V. N. Strocov, Entanglement and manipulation of the magnetic and spin-orbit order in multiferroic Rashba semiconductors, Nat. Commun. 7, 13071 (2016).

[13] J. H. Dil, Spin- and angle-resolved photoemission on topological materials, Electron. Struct. 1, 023001 (2019).

[14] J. Henk, K. Miyamoto, and M. Donath, Retrieving the initialstate spin polarization from spin-resolved photoemission: Proposal for a case study on W(110), Phys. Rev. B 98, 045124 (2018).

[15] D. Vasilyev, K. Medjanik, S. Babenkov, M. Ellguth, G. Schönhense, and H.-J. Elmers, Relation between spin-orbit induced spin polarization, Fano-effect and circular dichroism in soft x-ray photoemission, J. Phys.: Condens. Matter 32, 135501 (2019).

[16] D. Yu. Usachov, I. A. Nechaev, G. Poelchen, M. Güttler, E. E. Krasovskii, S. Schulz, A. Generalov, K. Kliemt, A. Kraiker, C. Krellner, K. Kummer, S. Danzenbächer, C. Laubschat, A. P. Weber, E. V. Chulkov, A. F. Santander-Syro, T. Imai, K. Miyamoto, T. Okuda, and D. V. Vyalikh, Observation of a cubic Rashba effect in the surface spin structure of rare-earth ternary materials, arXiv:2002.01701 [Phys. Rev. Lett. (to be published)].

[17] K. Koepernik and H. Eschrig, Full-potential nonorthogonal local-orbital minimum-basis band-structure scheme, Phys. Rev. B 59, 1743 (1999).

[18] See Supplemental Material at http://link.aps.org/supplemental/ 10.1103/PhysRevB.101.245140 for a derivation of equations, details of DFT calculations, and additional experimental and theoretical data.

[19] B. Chevalier, J. M. D. Coey, B. Lloret, and J. Etourneau, $\mathrm{EuIr}_{2} \mathrm{Si}_{2}: \mathrm{A}$ new intermediate mixed valence compound, J. Phys. C. Solid State Phys. 19, 4521 (1986).

[20] S. Seiro and C. Geibel, From stable divalent to valencefluctuating behavior in $\mathrm{Eu}\left(\mathrm{Rh}_{1-x} \mathrm{Ir}_{x}\right)_{2} \mathrm{Si}_{2}$ single crystals., J. Phys.: Condens. Matter. 23, 375601 (2011).

[21] S. Seiro, Y. Prots, K. Kummer, H. Rosner, R. C. Gil, and C. Geibel, Charge, lattice and magnetism across the valence crossover in $\mathrm{EuIr}_{2} \mathrm{Si}_{2}$ single crystals, J. Phys.: Condens. Matter 31, 305602 (2019).

[22] A. Chikina, M. Höppner, S. Seiro, K. Kummer, S. Danzenbächer, S. Patil, A. Generalov, M. Güttler, Yu. Kucherenko, E. V. Chulkov, Yu. M. Koroteev, K. Köpernik, C. Geibel, M. Shi, M. Radovic, C. Laubschat, and D. V. Vyalikh, Strong ferromagnetism at the surface of an antiferromagnet caused by buried magnetic moments, Nat. Commun. 5, 3171 (2014).

[23] M. Güttler, A. Generalov, M. M. Otrokov, K. Kummer, K. Kliemt, A. Fedorov, A. Chikina, S. Danzenbächer, S. Schulz, E. V. Chulkov, Yu. M. Koroteev, N. Caroca-Canales, M. Shi, M. Radovic, C. Geibel, C. Laubschat, P. Dudin, T. K. Kim, M. Hoesch, C. Krellner, and D. V. Vyalikh, Robust and tunable itinerant ferromagnetism at the silicon surface of the antiferromagnet $\mathrm{GdRh}_{2} \mathrm{Si}_{2}$, Sci. Rep. 6, 24254 (2016).

[24] A. Generalov, M. M. Otrokov, A. Chikina, K. Kliemt, K. Kummer, M. Höppner, M. Güttler, S. Seiro, A. Fedorov, S. Schulz, S. Danzenbächer, E. V. Chulkov, C. Geibel, C. Laubschat, P. Dudin, M. Hoesch, T. Kim, M. Radovic, M. Shi, N. C. Plumb, C. Krellner, and D. V. Vyalikh, Spin orientation of two-dimensional electrons driven by temperature-tunable competition of spin-orbit and exchange magnetic interactions, Nano Lett. 17, 811 (2017).

[25] M. Gmitra, A. Matos-Abiague, C. Draxl, and J. Fabian, Magnetic Control of Spin-Orbit Fields: A First-Principles Study of Fe/GaAs Junctions, Phys. Rev. Lett. 111, 036603 (2013).

[26] E. Arenholz, K. Starke, G. Kaindl, and P. J. Jensen, Interfacial Magnetism of Eu/Gd(0001) Studied by Magnetic Circular Dichroism in Photoemission, Phys. Rev. Lett. 80, 2221 (1998).

[27] K. Sakamoto, T. Oda, A. Kimura, K. Miyamoto, M. Tsujikawa, A. Imai, N. Ueno, H. Namatame, M. Taniguchi, P. E. J. Eriksson, and R. I. G. Uhrberg, Abrupt Rotation of the Rashba Spin to the Direction Perpendicular to the Surface, Phys. Rev. Lett. 102, 096805 (2009).

[28] L. V. Bondarenko, D. V. Gruznev, A. A. Yakovlev, A. Y. Tupchaya, D. Usachov, O. Vilkov, A. Fedorov, D. V. Vyalikh, S. V. Eremeev, E. V. Chulkov, A. V. Zotov, and A. A. Saranin, Large spin splitting of metallic surface-state bands at adsorbatemodified gold/silicon surfaces, Sci. Rep. 3, 1826 (2013).

[29] I. A. Nechaev and E. E. Krasovskii, Relativistic splitting of surface states at $\mathrm{Si}$-terminated surfaces of the layered intermetallic compounds $R T_{2} \mathrm{Si}_{2}$ ( $R=$ rare earth; $\left.T=\mathrm{Ir}, \mathrm{Rh}\right)$, Phys. Rev. B 98, 245415 (2018). 